\title{
Health impact assessments in London: assessing the London Mayoral strategies
}

\section{Caron Bowen}

Population Health Division, NSW Department of Health

Email:CBOWE@doh.health.nsw.gov.au

\begin{abstract}
Between 2001 and 2003 the London Health Commission undertook health impact assessments (HIAs) on a series of strategies developed by the Greater London Authority and the Mayor of London. The HIAs were rapid, each involving a literature review and a participant workshop. In all cases the reports made a series of recommendations that were given to the Mayor. The HIAs led to changes and ensured that health and health inequalities were given due consideration as part of the strategy development process.
\end{abstract}

The Mayor of London has responsibility for the development of several statutory strategies, including Economic Development, Transport, Spatial Development, Air Quality, Biodiversity, Municipal Waste Management, Ambient Noise and Culture. The London Health Commission undertook health impact assessments (HIAs) on each of the draft strategies and on several other non-statutory draft strategies the Mayor developed between 2001 and 2003. The process ensured that strategy proposals endeavoured to reduce health inequalities, mitigate negative health outcomes and increase positive health outcomes.

The HIAs were undertaken during the period in which the strategies were being scrutinised by the Greater London Assembly (the elected representatives of the Greater London Authority) before being released for public consultation. The HIA report formed part of the feedback that the Mayor and the strategy development teams considered before the document was circulated for consultation. The HIA was usually completed and the report submitted to the Mayor in between six and eight weeks, consequently the HIAs were rapid assessments.

\section{Screening}

There was no screening process as the HIAs had been agreed on by the Mayor and all of the strategies dealt with issues that could be considered determinants of health.
The steering group for each of the HIAs included representatives from a range of partner agencies with an interest in HIA, in health inequalities and in health outcomes. This included the London Health Commission, the London National Health Service Executive (now the Regional Public Health Group), the Greater London Authority and the London Health Observatory. The steering group also included the consultant responsible for the writing of the HIA report and, in some cases, members of the strategy development team.

For each HIA there were a number of stages. These were scoping, a literature review of the evidence, a stakeholder workshop, preparation of a draft report, presentation of the draft to the London Health Commission for review, then submission of the final report and recommendations to the Mayor and the Greater London Authority officers.

\section{Scoping}

Excepting the HIA for the London Plan, where a larger meeting was held, the steering group was responsible for scoping the HIA. The group met when the strategy was released to decide what should be addressed during the HIA and to develop tools for use in the stakeholder workshop. A public health specialist with knowledge of the health evidence related to the strategy area was engaged to undertake a literature review and to develop material to be presented to the participants of the workshop.

For each workshop, the steering group developed a workshop agenda and, in most cases, in partnership with the strategy development team, organised an invitation list. The agenda for the workshop focussed on specific areas and the literature review was structured to support participants in their discussions. Invitees included public health practitioners, local government employees, industry representatives and a range of interest groups and non-government organisations.

\section{Appraisal workshops}

The majority of the rapid appraisal workshops were halfday events. Each workshop began with a short introduction to the strategy and a presentation about the available evidence related to the health impacts that were to be considered. It was important to summarise the evidence ${ }^{1-3}$ as well as the main themes of the strategy to ensure that the workshop attendees had enough information to participate. Most of the workshop was spent in facilitated small groups, looking at specific areas of the strategy. During 
the early HIAs, the facilitator led the group through a series of general questions. As the HIA process developed, more detailed questions specifically related to the strategy were asked, enabling greater emphasis on health outcomes and health inequalities.

\section{The report}

After the workshop an HIA report was drafted using evidence from the workshop and the literature review. It was circulated to all the workshop participants for comment. The draft report and any comments received were then scrutinised by the steering group and the draft finalised. The report then went to the London Health Commission for debate and ratification and was submitted to the Mayor and the strategy development team.

The strategy development team was responsible for incorporating the recommendations and reporting back to the London Health Commission. Changes were made to the strategies as a result of recommendations (see Table 1 for examples ${ }^{4}$ ). The reporting to the London Health Commission was achieved through a presentation by the strategy development team explaining which recommendations had been accepted and, where recommendations had not been accepted, the reasons they had not been included in the redrafted version used for public consultation.

\section{Discussion}

Although the main reason for using the rapid HIA approach was the amount of time available for the process, there were advantages to using this method. It ensured that the HIA was part of the policy-making process ${ }^{5}$ but enabled the steering group to develop a timetable and work towards a completion date before the document went for public consultation. The workshops were scheduled and invitations sent out early to ensure that the maximum number of people invited were able to participate. Interest in the process grew as it became clear that the HIAs were influencing the strategies and for later HIAs there were, generally, greater numbers in attendance at the workshop. In addition, the use of published evidence to support recommendations was important, although the Greater London Authority was also interested in stakeholder opinion. In some cases, recommendations were made solely on the basis of stakeholder evidence due to a lack of evidence in the literature review. These issues have been discussed elsewhere. ${ }^{6}$

A disadvantage of holding only one workshop for each HIA and not undertaking interviews with stakeholders was that there was no opportunity for those unable to attend the workshop to participate in the process. This may have led to evidence and expert opinion not being considered. The steering group was also aware that there was no public involvement in the process. However, given the timing and resource issues it was not possible for there to be meaningful involvement and it was decided that stakeholder involvement would be more appropriate. $^{7}$

The London Health Commission commissioned an evaluation of the HIAs undertaken, which found that the use of HIA did have an impact on incorporating health considerations into the strategies. ${ }^{8}$

Table 1. Examples of the changes made to some of the London Mayoral strategies as a result of a health impact assessment

\begin{tabular}{ll}
\hline Strategy & Changes made \\
\hline Transport & - Increased emphasis on sustainable forms of transport \\
- Addition of a proposal to ensure that London Transport addresses the transport requirements of \\
groups with specific needs \\
- Increased emphasis on developing relationships with local government to ensure safety for \\
pedestrians \\
- Development of a walking and cycling plan for London \\
- Inclusion of a clear statement about the link between economic development and health \\
- Promoting the health of Londoners' incorporated into a new charter objective within the \\
strategy \\
- Revision of a charter objective promoting social inclusion and renewal for all of London's \\
communities \\
- Commitment by the London Development Agency to encourage and support breakfast clubs in \\
- London schools. \\
- Rddition of a proposal to work towards ensuring that Londoners are aware of the capital's green \\
- Recognition of the need to address perceived safety issues to encourage use of green spaces. \\
Biodiverstiy
\end{tabular}

Source: Bowen C. HIA and policy development in London: using HIA as a tool to integrate health considerations into strategy. In: Kemm J, Parry J, Palmer S, editors. Health Impact Assessment. Oxford: Oxford University Press, 2004. 


\section{References}

1. Curtis K, Roberts H. Children and health. Making the links. London: London Health Commission, 2003. Available from http://www.londonshealth.gov.uk/PDF/childrenandhealth.pdf

2. London Health Commission. Culture and health. Making the links. London: London Health Commission, 2003. Available from http://www.londonshealth.gov.uk/pdf/cultandh.pdf

3. London Health Commission. Noise and health. Making the links. London: London Health Commission, 2003. Available from http://www.londonshealth.gov.uk/pdf/noise_links.pdf

4. Bowen C. HIA and policy development in London: using HIA as a tool to integrate health considerations into strategy. In: Kemm J, Parry J, Palmer S, editors. Health Impact Assessment. Oxford: Oxford University Press, 2004.
5. Kemm J. Health Impact Assessment: a tool for healthy public policy. Health Prom Int 2001; 16(1): 79-85.

doi:10.1093/heapro/16.1.79

6. Mindell J, Boaz A, Joffe M, Curtis S, Birley M. Enhancing the evidence base for health impact assessment. J Epidemiol Community Health 2004; 58: 546-51. doi:10.1136/jech.2003.012401

7. Parry J, Stevens A. Prospective health impact assessment: pitfalls, problems and possible ways forward BMJ 2001; 323: 1177-82. doi:10.1136/bmj.323.7322.1177

8. London Health Commission. Evaluation of the Health Impact Assessments of on the draft Mayoral strategies for London. London: London Health Commission, 2003. Available from http://www.londonshealth.gov.uk/pdf/hiaeval.pdf 